\title{
The Effect of Proximal Lunate Morphology “Zapico Classification” on Kienböck's Disease
}

\author{
Davod Jafari, ${ }^{1}$ Hooman Shariatzadeh, ${ }^{1}$ and Bahman Hosseini ${ }^{1,}$ \\ ${ }^{1}$ Bone and Joint Reconstruction Research Center, Shafa Orthopedic Hospital, Iran University of Medical Sciences, Tehran, IR Iran \\ "Corresponding author: Bahman Hosseni, Bone and Joint Reconstruction Research Center, Shafa Orthopedic Hospital, Iran University of Medical Sciences, Tehran, IR Iran. Tel: \\ +98-2133542010, Fax: +98-2133542020, E-mail: bahman098@gmail.com
}

Received 2017 April 13; Revised 2017 June 16; Accepted 2017 July 06.

\begin{abstract}
Background: The Kienböck's disease (KD), even today, has many obscurities. Its etiologies as well as classifications are under constant change and debate, therefore we decided to define the effect of proximal lunate morphology on KD and its usefulness in evaluating the course of the disease.

Objectives: The purpose of this study is to compare the 3 different morphologies of the proximal lunate as defined by Antuna-Zapico (A-Z) in KD patients with a control group of normal individuals, and also compare the morphologies in different aspects like ulnar variance(UV), radial inclination and radial volar tilt, lunate sizes in radiological views, males and females, symptom duration before seeking medical treatment, lunate fragmentation, and also Lichtman's stages of the disease.

Methods: Unaffected wrist X-rays of $107 \mathrm{KD}$ patients (71 male 36 female), 17 - 53 years old, since 2011 to 2016, compared with 100 age and sex matched normal individuals divided in 3 lunate morphologies "as Antuna-Zapico has proposed"; noticing also the wrist indexes and the disease stages according to Lichtman's classification.

Results: The prevalence of 3 different A-Z classification morphologies were; 18 (16.8\%) Type1, 77 (72\%) Type 2, and 12 (11.2\%) Type 3 in the case group that wasn't statistically different from the control group, with 15 (15\%) Type 1, 73 (73\%) Type 2, and 12 (12\%) Type 3 (P $=0.9)$, even separately as male $(P=0.4)$ and females $(P=0.5)$. Case and controls were different in ulnar variance $U V,(P=0.001)$, however, different morphologies had a different $U V$ inside the case group $(P=0.4)$. All 3 morphologies were seen in all different Lichtman's stages with no correlation to the specific stage or morphology $(\mathrm{P}=0.2)$. In the case group, no correlation was found between different morphologies and lunate width $(\mathrm{P}=0.5)$, diameter $(\mathrm{P}=0.4)$, radial tilt $(\mathrm{P}=0.8)$, volar tilt $(\mathrm{P}=0.8)$.

Conclusions: Proximal lunate morphology, as depicted by A-Z, has no effect on the course of the disease and doesn't play any key role in the etiology. Its prevalence is the same as the general population.
\end{abstract}

Keywords: Lunate Morphology, Lunate Shape, Kienböck's Disease, Antuna-Zapico Classification, Lichtman's Stages

\section{Background}

After more than 100 years from the first description of avascular necrosis of lunate or osteomalacia of the lunate bone as Kienböck's disease KD (1), its cause still remains obscure and referred to as a multifactorial condition (2). Some authors have suggested that the morphology and microstructure of the wrist bones, intraosseous pressure, and mechanical stresses have etiologic and prognostic values (3-5). Ulnar variance (UV) is a good example of many author's attempt to show that the negative UV is playing a key role (4-15), despite many normal individuals with a negative UV, that doesn't get KD (6) and many KD patients with positive UV (16). One of the theories that has repeatedly been proposed is the effect of lunate morphology in $\mathrm{KD}$, either as an etiologic or a prognostic factor like Viegas and Zapico $(15,17,18)$. Antuna-Zapico (A-Z) theorized that the proximal lunate morphology is being seen in 3 different shapes and the first type is the weakest one that collapses very soon in the course of the disease and UV posi- tive is seen with type $3(17,18)$.

\section{Objectives}

There is a lot of information regarding KD, however, very little is written about lunate morphology and in this study we are defining the proximal lunate morphology, its effect on KD etiology, its course, as well as comparing it with normal individuals.

\section{Methods}

This retrospective study was performed after the review board approval of our institute. We evaluated the true posteroanterior (PA) and lateral (LAT) X-rays of uninvolved wrists of all KD patients diagnosed with an MRI and X-ray and treated in this center since 2011 till 2016.

Some cases were excluded because of missing desired data or concomitant ligamentous injury. Then, the age and 
sex matched group of random normal PA \& LAT wrist Xrays were obtained from the center's computer database with excluding any fractures, tumor, and ligamentous injury of the wrists or rheumatologic disease. Next, with marcopac's computer software, the digital calibrated images were measured and available analogue radiographs were evaluated considering and compensating the inherent magnification. With this we obtained 107 cases of KD patients between the ages of 17 - 53 years old with mean of $29 \pm$ SD 7.9 (59 right 48 left sides and 71 male 36 female). One hundred normal wrists of a normal population between 18 - 50 years old, with a mean of $32 \pm$ SD 7.7, with 64 male and 36 females were included as control group. First we divided all the cases to 3 different groups according to AZ1-3, type 1 with a trapezoid shape, type 2 rectangular, and type 3 pentagonal (7) (Figure 1). Then, we considered the age, sex, as well as the severity of the disease according to modified Lichtman's classification (Table 1), and the delay in seeking the treatment and radial tilt or inclination [as in PA radiographs the angle between the line drawn through the distal most radio carpal articular surface to distal most radio ulnar joint's radial side with the line perpendicular to the ulnar shaft or radial mid shaft], volar tilt [as in LAT radiographs the angle between the line drawn through the volar and dorsal distal most lips of distal radius articular surface and the radial shaft], and ulnar variance UV [as in PA radiographs, distance between distal most ulnar articular surface and distal most radial articular surface plateau in ulnar side]. We studied the affected sides for having a visible coronal fracture in the LAT X-rays. The lunate diameter in both radiologic PA and LAT views were measured in the unaffected side radiographs. In the LAT view we measured the biggest dimensions of the lunate volar to dorsal diameter. In the PA view, due to fact that the lunate dimensions are smaller at distal and bigger at proximal, the lunate diameter was measured from the middle of its both medial and lateral sides. This means that in the PA view, it is a line connecting 2 points of middle of scapholunate and lunatotriquetral joints (Figures 2 and 3). Statistical analysis was performed using IBM SPSS for windows, version 21. P value of $<0.05$ was considered as significant.

\section{Results}

There were 18 (16.8\%) Type 1,77 (72\%) Type 2, and 12 (11.2\%) Type 3 in the case group and 15 (15\%) Type 1, 73 (73\%) Type 2, and $12(12 \%)$ Type 3 in the control group. Comparing the case and control group showed that the prevalence of 3 different shapes in cases and control group is not statistically different $(\mathrm{P}=0.9)$. This was also the same for males $(\mathrm{P}=$ 0.4 ) and females $(P=0.5)$, respectively. In comparing the different stages of the disease, according to the Lichtman's
Table 1. Lichtman Classification for the Stages of the Kienböck's Disease

\begin{tabular}{ll}
\hline Stages & Definition \\
\hline $\mathbf{1}$ & $\begin{array}{l}\text { Plain radiographs are normal MRI shows diffuse t1 signal decrease } \\
\text { positive scintigraphy }\end{array}$ \\
\hline $\mathbf{2}$ & Sclerosis of lunate with no collapse of bone \\
\hline $\mathbf{3 A}$ & Lunate collapse with carpal height preserved \\
$\mathbf{3 B}$ & Lunate collapse with hyper flexed scaphoid \\
\hline $\mathbf{4}$ & Carpal arthritis \\
\hline
\end{tabular}

classification, in the case group there were no correlations between severity of the disease and the lunate morphology with post hoc multiple comparison $(\mathrm{P}=0.2)$. The 3 different morphologies showed no correlation with lunate width in PA X-ray $(\mathrm{P}=0.5)$ and lunate diameter in LAT X-ray $(\mathrm{P}=0.2)$. There has also been no correlation between these figures and the symptom duration (mean 15 months) before seeking medical treatment $(\mathrm{P}=0.5)$. There has been no correlation between these figures and fragmentation of the lunate visible in LAT $\mathrm{x}$-ray views $(\mathrm{P}=0.6)$, radial $(\mathrm{P}=0.8)$, and volar tilt $(\mathrm{P}=0.8)$. However, strong correlation was found for the UV difference in the case and control groups ( $\mathrm{P}=$ 0.001), (Figure 4). No difference was found in UV between morphologies in the case group $(\mathrm{P}=0.4)$.

\section{Discussion}

A-Z tried to prove that the lunate morphology is related to ulnar variance and theorized that molding of lunate with aging as well as grip and dominancy could be responsible for the lunate shape during growth. It means that ulnar plus variance is the cause of A-Z type 3 lunate. However, like other studies (14), we did not find a correlation between ulnar Variance and lunate type. We could not find any relationship of UV and A-Z lunate morphologies.

Another matter of debate is the shape defined by A-Z, which is somewhat obscure; it means there are many lunates with a tiny knob in the middle of proximal lunate joint that doesn't fit to the definition of types. Furthermore, defining the angle in scaphoid side between 2 curves is prone to miscalculations. To overcome the sampling bias we matched the groups in regard to age and sex. Tsuge and Nakamura (8) compared the result of 41 normal sides of KD with 66 normal populations and showed smaller lunate and flatter radially inclined distal radius that we couldn't prove such relationship. Lamas et al. (9), in cadaveric study, showed $20.8 \%$ Type 1, 75\% Type 2, and $4.2 \%$ Type 3 , that nearly matches our series. However, smaller lunate in Type 1 as well as the nut cracker effect to have more fragmentation and ulnar minus in Type 1 and the weaker Type 


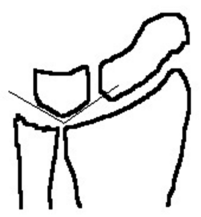

Type3 Pentagonal

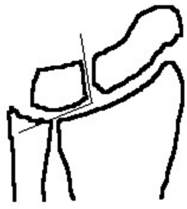

Type2 Rectangular
Angle $<110$

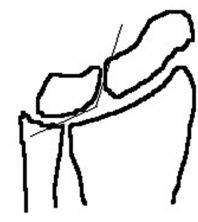

Type 1 Trapezo
Angle 130

Figure 1. Antuna-Zapico Types 1- 3 of Lunate Bone Morphology

Figure 2. In PA View X Ray

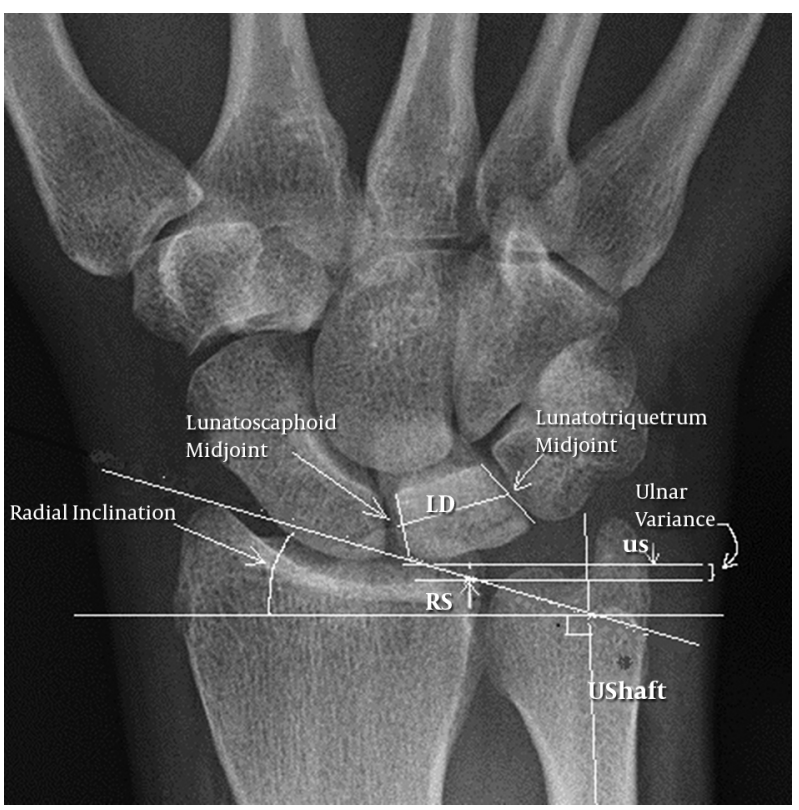

U Shaft, ulnar shaft; US, ulnar surface; LD, lunate diameter; RI, radial inclination; RS, radial surface.

1 because of bony structure as perpendicular trabecular to the surfaces $(10,12)$ which were not repeated in our study.

Ledoux et al. (11) proposed that ulnar minus, lunate uncovering, and angulated trabecular cause the micro fractures not to heal properly. Garcia-Elias and Vidal (13), in a group of KD 28 men and 12 women, connected the lunate length to lunate morphology but assumed some exceptions, unlike the Schuurman et al. (14) with 68 wrists with no connection of ulnar length to lunate shape. UV difference between case and control group in our study is similar to the reports that are in favor of ulnar minus having a role in $\mathrm{KD}$ (4) and against those reports who believe that UV has no role in $\mathrm{KD}(6)$.
Figure 3. In LAT View X Ray

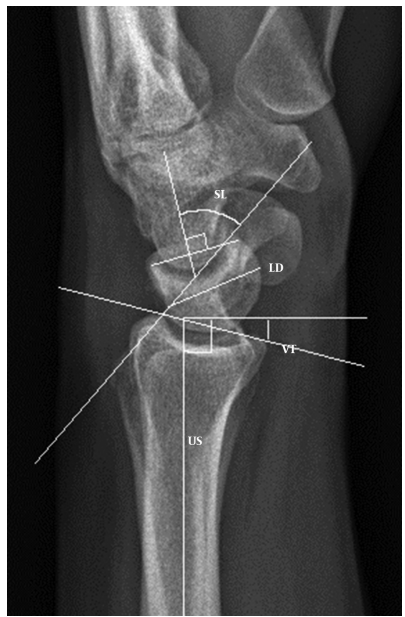

US, ulnar shaft; VT, volar tilt; LD, lunate diameter; SL, scaphoid lunate angle.

\subsection{Conclusions}

It seems that proximal lunate morphology as A-Z classification has no effect in differing the course of the KD, is not affected by carpal indexes, has the same prevalence in KD and normal population, and we didn't find the molding effect of distal ulna.

\section{Acknowledgments}

We thank Dr. Bahardoust for his valued help with the statistical analyzing. We would also like to thank Mrs. Razavian for providing the required data from the radiological ward computer's database.

\section{Footnote}

Author's Contribution: Study design and supervision: Davod Jafari; data collection: Hooman Shariatzadeh, Bahman Hosseni; manuscript drafting and revision: Davod Jafar, Hooman Shariatzadeh, Bahman Hosseni. 
Figure 4. Difference in UV in Case and Controls in a X CHART with Shown Frequency of Each One

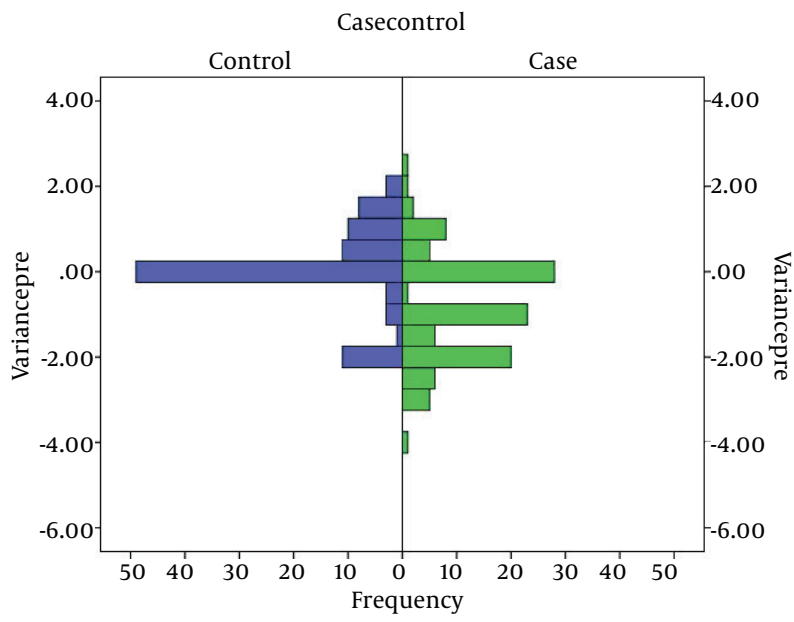

Variancepre, pre-operative UV.

\section{References}

1. Wagner JP, Chung KC. A Historical Report on Robert Kienböck (1871-1953) and Kienböck's Disease.J Hand Surg. 2005;30(6):1117-21. doi: 10.1016/j.jhsa.2005.08.002.

2. Lichtman DM, Lesley NE, Simmons SP. The classification and treatment of Kienbock's disease: the state of the art and a look at the future. J Hand Surg. 2010;35(7):549-54.

3. Schiltenwolf M, Martini AK, Mau HC, Eversheim S, Brocai DR, Jensen $\mathrm{CH}$. Further investigations of the intraosseous pressure characteristics in necrotic lunates (Kienbock's disease). $J$ Hand Surg Am. 1996;21(5):754-8. doi: 10.1016/S0363-5023(96)80187-0. [PubMed: 8891969].
4. Goeminne S, Degreef I, De Smet L. Negative ulnar variance has prognostic value in progression of Kienbock's disease. Acta Orthop Belg. 2010;76(1):38-41. [PubMed: 20306962].

5. Han KJ, Kim JY, Chung NS, Lee HR, Lee YS. Trabecular microstructure of the human lunate in Kienbock's disease. J Hand Surg Eur Vol. 2012;37(4):336-41. doi: 10.1177/1753193411422337. [PubMed: 21965181].

6. D'Hoore K, De Smet L, Verellen K, Vral J, Fabry G. Negative ulnar variance is not a risk factor for Kienböck's disease. J Hand Surg. 1994;19(2):229-31. doi: 10.1016/0363-5023(94)90010-8.

7. Rhee PC, Jones DB, Moran SL, Shin AY. The effect of lunate morphology in Kienbock disease. J Hand Surg Am. 2015;40(4):738-44. doi: 10.1016/j.jhsa.2014.12.024. [PubMed: 25701489].

8. Tsuge S, Nakamura R. Anatomical risk factors for Keinböck's disease.J Hand Surg. 1993;18(1):70-5.

9. Lamas C, Carrera A, Proubasta I, Llusa M, Majo J, Mir X. The anatomy and vascularity of the lunate: considerations applied to Kienböck's disease. Chirurgie de la main. 2007;26(1):13-20.

10. Viegas SF, Patterson RM, Todd PD, McCarty P. Load mechanics of the midcarpal joint. J Hand Surg Am. 1993;18(1):14-8. doi: 10.1016/03635023(93)90238-X. [PubMed: 8423300].

11. Ledoux P, Lamblin D, Wuilbaut A, Schuind F. A finite-element analysis of Kienböck's disease. J Hand Surg. 2008;33(3):286-91.

12. Iwasaki N, Genda E, Minami A, Kaneda K, Chao EYS. Force transmission through the wrist joint in Kienböck's disease: a two-dimensional theoretical study. J Hand Surg. 1998;23(3):415-24.

13. Garcia-Elias M, Vidal AM. Kienbock's disease. Curr Orthopaed. 1997;11(1):28-35.

14. Schuurman H, Maas M, Dijkstra PF, Kauer JMG. Ulnar variance and the shape of the lunate bone. A radiological investigation. Acta orthopaedica belgica. 2001;67(5):464-7.

15. Viegas SF, Wagner K, Patterson R, Peterson P. Medial (hamate) facet of the lunate. J Hand Surg. 1990;15(4):564-71.

16. Nakamura R, Imaeda T, Miura T. Radial shortening for Kienböck's disease: factors affecting the operative result. J Hand Surg. 1990;15(1):405.

17. Antu-a-Zapico JM. Enfermedad de Kienbock. Revista de ortopedia y traumatologia. Orthopaedicsand Trauma Journal. 1993;37:100-13.

18. Antu-a-Zapico JM. Malacia del semilunar. University of Valladolid; 